\title{
Editorial
}

\section{Statistical methods in medical research}

\author{
Ganeshkumar P*
}

\author{
Department of Community Medicine, SRM Medical College Hospital \& Research Centre, SRM University \\ Kattankulathur, Tamil Nadu, India
}

\author{
* Correspondence: \\ Dr. Ganeshkumar P, \\ E-mail: ganeshkumardr@gmail.com
}

\begin{abstract}
Medicine is an ever changing science. Thus new knowledge generated by research and clinical experience widen the knowledge; change the understanding of natural history of disease and its application in therapeutics. Thus policy makers, clinicians and researchers must evaluate and use information existing in the literature to implement in their healthcare delivery. The father of field epidemiology, John Snow during 1854 studied the cholera epidemic in London and demonstrated the association of epidemiological and statistical methods in medical research. Popularity gained after Bradford Hill's lectures were published as a series of articles in the Lancet and then in book form, principles of medical statistics. ${ }^{1}$ The book went through 11 editions during his life-time.
\end{abstract}

Medical science is dynamic and implementation of its facts is based on observational science where hypotheses are generated from known and unknown areas. Statistics provides sound methods in observing the health related events in collecting the data, techniques to summarize \& analyze so as to draw valid inferences regarding the hypothesis. Statistics can be used for comparison as well as to study the relationship between two or more factors. The use of such relationship further helps to predict one factor from the other.

Statistics are broadly classified into two categories, Descriptive and Inferential statistics. Descriptive statistics deals with simplification of the data into understanding form to give concise description of complex information and demonstrating the pattern in the data sets. As medical research and clinical observation never stops at description, it tends to determine whether the characteristics and pattern in data sets are unusual or happened by chance. This step is crucial in taking decisions and application of the findings into clinical application. Inferential statistics helps in taking these decisions which includes hypothesis testing.
As variability \& uncertainty are the key issues in medical science, statistics helps in taking decisions from data collected with variability. Thus statistical methods are vital in creating solutions for this complexity. Biostatistics has developed enormously in recent years, due to continuing advances in diverse biomedical areas and fields. For example, new problems in biomedical research have led to the development of new statistical methodologies that would not otherwise have arisen, and at the same time have favoured ingenious adaptations of classical statistical techniques to new contexts of application. $^{2,3}$

The link between biostatistics and epidemiology has constantly been close. Earliest epidemiologist used certain statistical methods much on infectious diseases in the study of epidemics and evaluation of therapies. Those research methods were prospective which became impracticable to study the chronic diseases. Primarily Cornfield and Mantel provided the basis of valid inferences from case control data which began in exploring models such as dose response models - for evaluating the effects of possible risk factors for disease. This led to the development of probabilistic concepts such as the odds ratio or relative risk as measures of association.

Clinical trials are an indispensable part of the medical research because through clinical trials, scientific finding can lead to improved ways of preventing, detecting, and treating diseases and clinical scenarios. Recent years have seen a major raise in the significance of statistics in the field of drug development. Statistics acts a critical part at all stages of the clinical trial, from planning, through conduct and interim analysis, to final analysis and reporting. The statistician will typically formulate the randomization schedules, recommend on sample size, indicate criteria for measuring treatment differences, and analyze response rates. 
Persistent issues in clinical trials insist the continuing development of statistical methods for handling subgroups in the design and analysis of clinical trials. The involvement of statisticians with different perspectives continues to enrich quality of research and its findings. The domains of statistics that have influenced more recently are generalized linear models (including multiple linear regression), survival analysis, categorical data analysis, spatial statistics, and Bayesian methods (in diagnostic, epidemiological and clinical trials contexts). According to Altman and Goodman the new statistical methods playing a key role in biomedical research over coming years are bootstrap (and other computer-intensive methods), Gibbs sampler (and other Bayesian methods), generalized additive models, Classification And Regression Trees (CART), models for longitudinal data and hierarchical data \& neural networks. Meta-analysis, as a tool for evidence-based medicine, has attracted substantial consideration in recent years which is based on statistical techniques to derive a summary estimate or statement from multiple research studies. Randomized Clinical Trials (RCTs) actually play a significant role in meta-analysis through generation of evidence which in turn lead on to knowledge transfer / translational research and practice.
Due to advent of computer and statistical software in current era complex statistical technique can be applied easily in different clinical and research contexts. However due to the lacunae in capacity among the researchers and unavailability of the standardized software limits application of these techniques for the current clinical requirement. Hence immense application of statistical methods in the healthcare research, training and clinical services ensure the improved quality of healthcare delivery and assured patient safety.

\section{REFERENCES}

1. Hill AB. Principles. In: Hill AB, eds. Principles of Medical Statistics. 1st ed. London: Lancet, Oxford University Press; 1937:189.

2. DeMets DL, Stormo G, Boehnke M, Louis TA, Taylor J, Dixon D. Training of the next generation of biostatisticians. Stat Med. 2006 Oct;25(20):341529.

3. Zelen M. Biostatisticians: biostatistical science and the future. Stat Med. 2006 Oct;25(20):3409-14.

DOI: $10.5455 / 2349-3259$. ijct20140501

Cite this article as: Ganeshkumar P. Statistical methods in medical research. Int J Clin Trials 2014;1:1-2. 